\title{
Congenital heart disease protein 5 associates with CASZ1 to maintain myocardial tissue integrity
}

\author{
Stephen Sojka ${ }^{1,2}$, Nirav M. Amin ${ }^{1,3}$, Devin Gibbs ${ }^{2}$, Kathleen S. Christine ${ }^{1,2}$, Marta S. Charpentier ${ }^{1,3}$ and \\ Frank L. Conlon ${ }^{1,2,3, *}$
}

\begin{abstract}
The identification and characterization of the cellular and molecular pathways involved in the differentiation and morphogenesis of specific cell types of the developing heart are crucial to understanding the process of cardiac development and the pathology associated with human congenital heart disease. Here, we show that the cardiac transcription factor CASTOR (CASZ1) directly interacts with congenital heart disease 5 protein (CHD5), which is also known as tryptophanrich basic protein (WRB), a gene located on chromosome 21 in the proposed region responsible for congenital heart disease in individuals with Down's syndrome. We demonstrate that loss of CHD5 in Xenopus leads to compromised myocardial integrity, improper deposition of basement membrane, and a resultant failure of hearts to undergo cell movements associated with cardiac formation. We further report that CHD5 is essential for CASZ1 function and that the CHD5-CASZ1 interaction is necessary for cardiac morphogenesis. Collectively, these results establish a role for CHD5 and CASZ1 in the early stages of vertebrate cardiac development.
\end{abstract}

KEY WORDS: Heart morphogenesis, Proliferation, Morphogenesis, Heart development, Cardiac, Congenital heart disease, Down's syndrome, Xenopus, CASTOR, CASZ1, CHD5, WRB, Cardiomyocyte

\section{INTRODUCTION}

Congenital heart disease is an endemic disorder occurring in 1\% of all live births (Roger et al., 2011). Down's syndrome (DS) is the most common cause of human genetic heart defects with an estimated $35-60 \%$ of individuals with DS afflicted with a range of cardiac abnormalities, most frequently including atrioventricular septal defects, atrial and ventricular septal defects, tetralogy of Fallot, and patent ductus arteriosis (Goodship et al., 1998; Chaoui et al., 2005; Abbag, 2006). The diversity of cardiac phenotypes along with the inheritance of varying regions of an extra copy of chromosome 21 , as well as additional chromosomal abnormalities in a subset of individuals with DS, has made identifying the crucial gene(s) for heart disease in DS patients problematic (Korbel et al., 2009; Lyle et al., 2009). Although recent studies have used mouse models to identify the putative crucial region in chromosome 21 that is associated with heart disease in DS patients, specific genes or groups of genes responsible for any of the cardiac phenotypes have not been identified (Shinohara et al., 2001; Liu et al., 2011a). Among the candidate genes is that

\footnotetext{
${ }^{1}$ University of North Carolina McAllister Heart Institute, UNC-Chapel Hill, Chapel Hill, NC 27599-3280, USA. ²Department of Biology, UNC-Chapel Hill, Chapel Hill, NC 27599-3280, USA. ${ }^{3}$ Department of Genetics, UNC-Chapel Hill, Chapel Hill, NC 27599-3280, USA.

*Author for correspondence (frank_conlon@med.unc.edu)
}

Received 27 November 2013; Accepted 29 May 2014 encoding congenital heart disease protein 5 (CHD5), also known as tryptophan-rich basic protein (WRB). CHD5, initially identified in a screen for genes within a restricted region of chromosome 21 associated with heart disease in individuals with DS, was found to be expressed in fetal hearts and shown to localize predominately to the nuclei of cells of cardiac origin (Egeo et al., 1998). CHD5 was also identified in a drug-sensitized screen in zebrafish as a regulator of myocardial repolarization (Milan et al., 2009) and depletion of CHD5 in medaka fish has been reported to be associated with general cardiac defects of unknown etiology (Murata et al., 2009). In addition to these findings, CHD5 has also been reported to be localized to the membrane of the endoplasmic reticulum (ER), where it is proposed to function as a receptor to transport charged proteins across the ER membrane (Ando and Suzuki, 2005; Vilardi et al., 2011). Currently, no CHD5 knockout mouse exists, so the cellular requirements and the mechanisms by which CHD5 functions in vertebrates remain to be established.

Studies investigating the transcriptional control of cardiomyocyte differentiation have revealed a fundamental requirement for the para-zinc-finger transcription factor CASTOR (CASZ1) in vertebrate heart development. Depletion of CASZ1 in Xenopus embryos results in failure of a subset of progenitor cells to differentiate into cardiomyocytes, resulting in cardia bifida and aberrant cardiac morphogenesis (Christine and Conlon, 2008). To date, there have been limited studies on CASZ1 in mammals; however, a partial murine Casz1 cDNA was shown to be expressed in the primitive heart tube in mice (Vacalla and Theil, 2002) and Caszl was shown to be expressed in adult human heart tissue (Liu et al., 2006). Intriguingly, two recent genome-wide association studies (GWAS) showed a genetic association between the human Caszl locus and high blood pressure and hypertension (Levy et al., 2009; Takeuchi et al., 2010), implying a potential link between CASZ1 and cardiovascular dysfunction. Consistent with this, it has been recently demonstrated that CASZ1 has an essential and evolutionarily conserved role in blood vessel assembly and lumen formation (Charpentier et al., 2013a,b).

To determine the molecular mechanisms by which CASZ1 functions to control cardiac morphogenesis, we conducted a two hybrid screen and identified CHD5 as a CASZ1-interacting partner. We have gone on to demonstrate that CASZ1 and CHD5 are colocalized in the nuclei of the developing myocardium. We further show that CHD5 is essential for cardiomyocyte adhesion and basement membrane depositions, and that loss of CHD5 leads to a failure of cardiomyocytes to undergo the appropriate cell movements associated with the formation of a properly looped heart. Finally, we show that CHD5 is essential for CASZ1 function and the CHD5-CASZ1 interaction is necessary for cardiac morphogenesis. Collectively, these studies establish a role for CASZ1 and CHD5 for maintaining cardiomyocyte integrity. 


\section{RESULTS}

\section{CASZ1 interacts with CHD5}

It is well established that the molecular mechanisms of early heart development are highly conserved from Xenopus to human (Brade et al., 2007; Goetz and Conlon, 2007; Warkman and Krieg, 2007; Bartlett and Weeks, 2008; Afouda and Hoppler, 2009; Gessert and Kuhl, 2009; Evans et al., 2010; Kaltenbrun et al., 2011; Tandon et al., 2013), and studies in Xenopus have demonstrated an essential role for the transcription factor CASZ1 in cardiac differentiation and heart morphogenesis (Christine and Conlon, 2008). To ascertain the molecular mechanism by which CASZ1 functions in cardiac development, we undertook a yeast two-hybrid screen, using fulllength Xenopus CASZ1 as bait to screen a cDNA library that we generated from stage 28 Xenopus cardiac enriched tissue, a period that approximately corresponds to E7.5 in mouse and $18 \pm 1$ days in human. Thus, the library was created from tissue at the time and place when CASZ1 is required (Christine and Conlon, 2008). It was deemed 'cardiac enriched' because at this period of development heart tissue is contiguous with lateral mesoderm and, therefore, we cannot state that we isolated heart tissue exclusively. From this screen, we identified the 33 most C-terminal residues (amino acids 137-170) of Xenopus CHD5 (Fig. 1A,B). CHD5 is a coiled-coil $14 \mathrm{kDa}$ protein initially cloned and identified from the minimal chromosomal region associated with congenital heart disease in individuals with DS (Fig. 1C). Accordingly, it has been shown that CHD5 is predominantly expressed within the nuclei of cultured human fibroblasts derived from fetal endocardial tissue (Egeo et al., 1998). We identified a putative Xenopus CHD5 EST and confirmed the clone to be full length (Fig. 1C; supplementary material Fig. S1A,E). Subsequent analysis by BLAST and Metazome (www.metazome. net) demonstrated the clone to be the homolog of CHD5 from zebrafish, chicken, mouse and human, as it has similar exon/intron structure and is syntenic to other CHD5 homologs (supplementary material Fig. S1A-E). Examination of the region of CHD5 that interacts with CASZ1 (amino-acids 137-170) showed CHD5 to be $76 \%$ identical and $94 \%$ similar to the human CHD5 ortholog (supplementary material Fig. S1B). We further demonstrated that CASZ1 and CHD5 interact via co-immunoprecipitation of epitopetagged proteins in Xenopus embryos, confirming that CASZ1 and CHD5 can interact in vivo (supplementary material Fig. S1F).

RT-PCR revealed CHD5 to be expressed at all stages of development (Fig. 1D) and, in agreement with CHD5 being identified as an interacting partner of CASZ1, Chd5 was found to be expressed at low levels in cardiac tissue, as well as at higher levels in anterior mesoderm and neural tissue of early Xenopus tadpoles (Fig. 1E; supplementary material Fig. S2). We found that CHD5 was expressed ubiquitously throughout the developing heart, and that CHD5 and CASZ1 colocalized in the developing myocardium within the nuclei of cardiomyocytes (Fig. 1F-M; supplementary material Fig. S3). Since it has been established in published studies that CASZ1 is expressed in the myocardium (Christine and Conlon, 2008; Amin et al., 2014), these findings demonstrate that CHD5 and CASZ1 are co-expressed within the developing myocardium and physically associate.

\section{CHD5 is required for cardiac morphogenesis}

The observation that $C h d 5$ is contained within the genomic region mutated in DS patients with congenital heart disease and the observation that CHD5 interacts with CASZ1 imply a role for CHD5 in heart development. To test this hypothesis, we depleted Chd5 in Xenopus embryos using translation blocking and spliceblocking antisense morpholinos (MOs) (supplementary material Figs S4-S6). We did not observe any overt phenotypes prior to early tadpole stages (stage 29 and 32), when linear heart tube formation

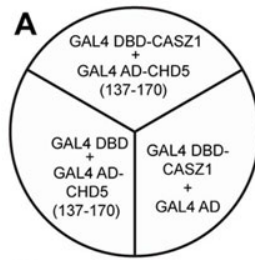

D
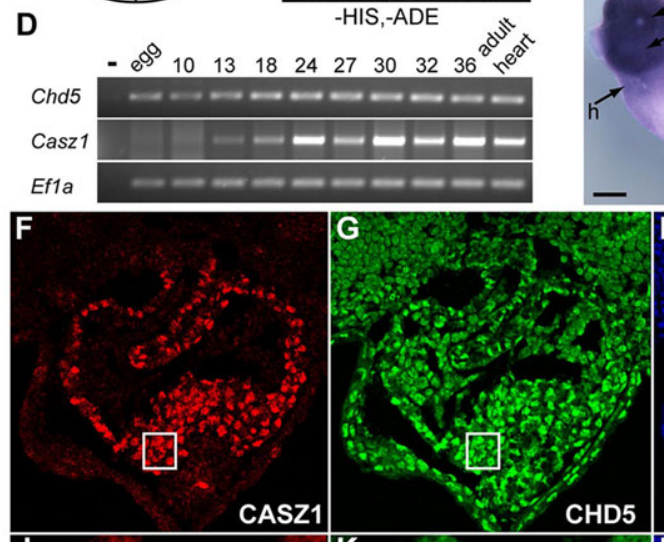

C $\mathrm{CHD} 5$
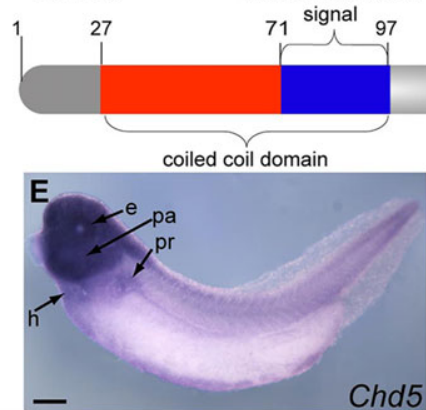

nuclear localization $71 \overbrace{}^{\text {signal }} 97$

Chd5
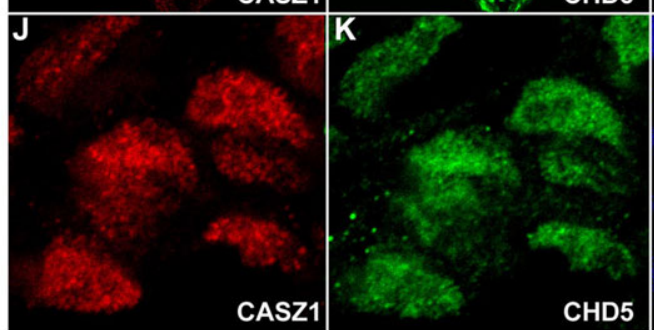
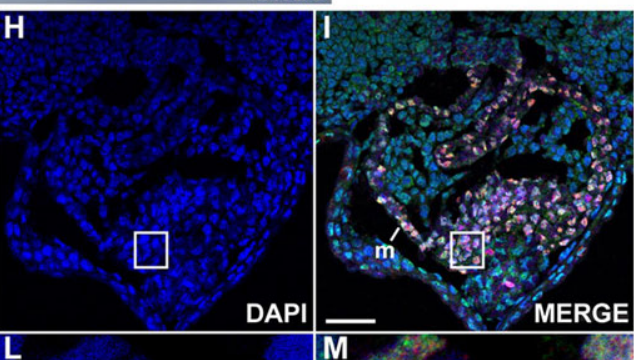

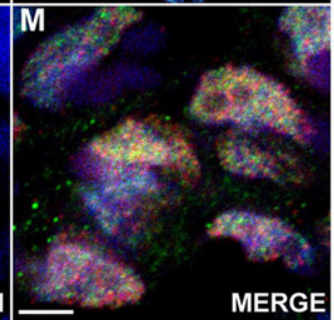

Fig. 1. CASZ1 interacts with CHD5.

$(A, B)$ Full-length CASZ1 (bait) interacts with a partial CHD5 protein (prey) in a yeast two-hybrid assay. Cells placed under-His/-Ade selection. (C) Schematic of the structure of CHD5 depicting predicted domains. (D) RT-PCR on cDNA derived from Xenopus whole-embryo lysate. Ef1a was used as a loading control. (E) Expression of Chd5 RNA by in situ analysis in early tadpole Xenopus embryos (stage 37). h, heart; pr, pronephros; e, eye; pa, pharyngeal arches. (F-M) Colocalization of CHD5 and CASZ1 protein in the nuclei of the developing Xenopus (stage 40) myocardium $(\mathrm{m})$. White boxes in $\mathrm{F}-\mathrm{I}$ correspond to the magnified regions in J-M. Scale bars: $500 \mu \mathrm{m}$ in E; $50 \mu \mathrm{m}$ in F-l; $5 \mu \mathrm{m}$ in $\mathrm{J}-\mathrm{M}$. 

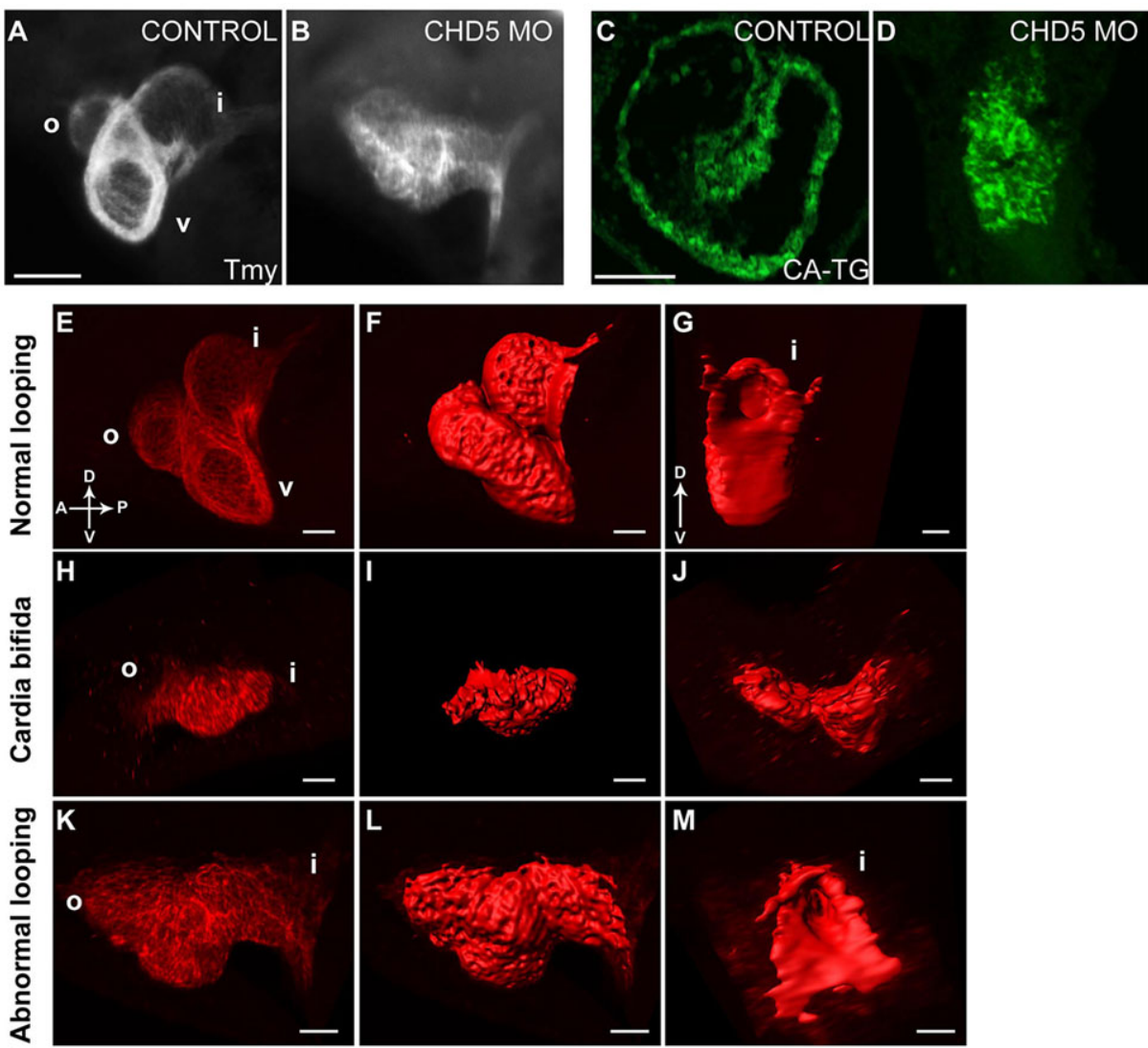

Fig. 2. CHD5 is required for cardiac morphogenesis. (A,B) CHD5-depleted embryos (stage 37 ) exhibit smaller, improperly looped hearts that fail to undergo chamber formation. Lateral views of whole-mount antibody staining with anti-tropomyosin (Tmy) antibody. (C,D) CHD5-depleted hearts fail to develop fully formed chambers and have a thicker myocardial layer. Transverse sections of cardiac actin-EGFP transgenic (CA-TG) Xenopus embryos (stage 37). (E-M) 3D reconstructions of early tadpole Xenopus embryos (stage 37) stained with antitropomyosin antibody viewed laterally and posteriorly. CHD5-depleted hearts (H-M) fail to complete migration and fusion $(\mathrm{H}-\mathrm{J}, 54 \%)$, or fail to undergo proper looping and chamber formation (K-M, 31\%). Only 15\% display normal looping. $n>25$ per condition, two biological replicates. $v$, ventricle; o, outflow tract; i, inflow tract. D, dorsal; A, anterior; $\mathrm{V}$, ventral;

$P$, posterior. Scale bars: $100 \mu \mathrm{m}$ in A-D; $50 \mu \mathrm{m}$ in E-M. and cardiac looping begins, using either splice-blocking or translational-blocking MOs (supplementary material Fig. S7). Consistently, we did not detect alterations in the expression of the cardiac markers $N k \times 2.5$ and $T b \times 20$, collectively indicating that CHD5 is not essential for cardiac specification (supplementary material Fig. S7A-X).

By cardiac-looping stages (stage 37) (Newman and Krieg, 1998; Kolker et al., 2000; Mohun et al., 2000), we observed overt alterations in CHD5-depleted hearts relative to controls. Examination of cardiac tissue by tropomyosin expression showed that CHD5-depleted hearts initiated differentiation but failed to complete the processes of cardiac looping and chamber formation (Fig. 2A,B). Consistently, transverse sections of cardiac actinEGFP transgenic embryos (CA-TG) (Latinkic et al., 2002) showed that, in comparison with the distinct cardiac chambers of control embryos, CHD5-depleted embryos displayed an underdeveloped and thickened tubular linear heart tube (Fig. 2C,D). The cardiac defects did not appear to be secondary to broader defects in anterior patterning as we did not note discernible differences in expression of anterior markers Otx2 (Pannese et al., 1995) and Eomes (Ryan et al., 1998) between control and CHD5-depleted embryos (supplementary material Fig. S8).

To gain insight into the underlying cellular defects of CHD5depleted hearts, we performed $3 \mathrm{D}$ reconstruction on Z-stack images of tropomyosin-stained hearts (Fig. 2E-M; supplementary material Movies 1 and 2). In contrast to control hearts (Fig. 2E-G; supplementary material Movie 1), CHD5-depleted hearts either arrested cell movements prior to fusion of the cardiac fields at the midline (Fig. 2H-J, 54\%) or disrupted cell movements prior to chamber formation (Fig. 2K-M, 31\%; supplementary material Movie 2). Ultrastructural analysis performed by scanning electron microscopy (SEM) on hearts from cardiac looping stages (stage 37) revealed that (regardless of the class of phenotype) the majority of CHD5-depleted embryos contained cardiac tissue that failed to undergo the normal cell shape changes associated with development and maturation of the linear heart tube (Fig. 3A-D). We further observed a failure of looping and chamber formation in CASZ1-depleted embryos (Fig. 3E,F), and that the CHD5-depleted hearts had a more severe phenotype than those derived from CASZ1-depletion, suggesting that CHD5 may function by interacting with other cardiac proteins. However, we found that in both CHD5- and CASZ1-depleted hearts the myocardial tissue remained as a loose collection of cells versus controls (Fig. 3A,B,E).

Further examination showed that the alteration in cardiac morphology in CHD5-depleted hearts was associated with a decrease in cardiomyocyte cell number (mean $=1817 \pm 130$ versus $1499 \pm 184, n \geq 3$ ) and a lower mitotic index than stage-matched controls (mean $=2.98 \pm 0.68$ versus $1.07 \pm 0.42, n \geq 3$ ), but not with an increase in cell death (supplementary material Fig. S9). Thus, CHD5-depleted cardiomyocytes remain viable and undergo cell division albeit at a slower rate.

\section{CHD5 and CASZ1 are required for cardiomyocyte adhesion and deposition of basement membrane}

Our findings that CHD5- and CASZ1-depleted hearts appear as a loose group of myocardial tissue versus the smooth surface of control hearts led us to investigate the tight junction marker zonula occludens-1 (ZO-1) which is expressed at cell-cell contacts in cardiomyocytes at cardiac-looping stages (stage 37). In stark contrast to control embryos, ZO-1 expression is either diffuse or absent in CHD5 and CASZ1-depleted myocardial tissue (Fig. 4A-L). These findings are corroborated by transmission electron microscopy (TEM) which shows that at the cardiac-looping stage (stage 37) depletion of either CHD5 or CASZ1 is associated with large gaps 

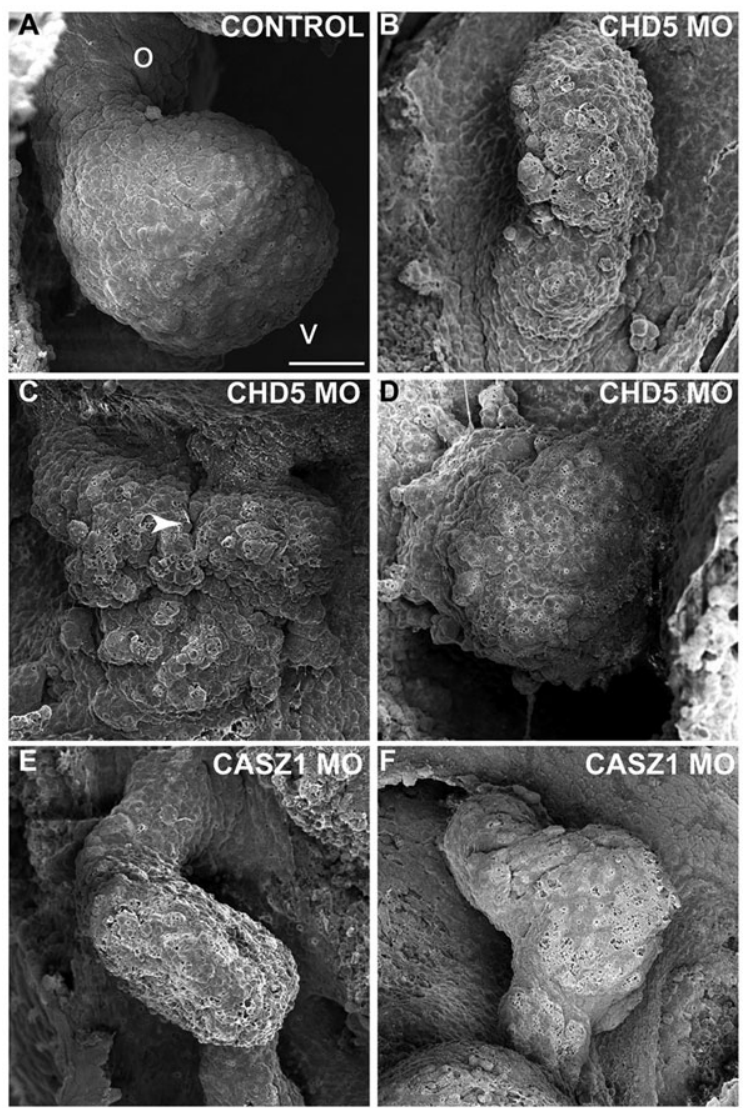

Fig. 3. CHD5 is required for cardiac morphogenesis. (A-D) Scanning electron microcopy (SEM) of Xenopus embryos at early tadpole stage (stage 37) in (A) control and (B-D) CHD5-depleted embryos. CHD5-depleted embryos fail to complete looping and begin chamber formation, and fail to undergo normal cell shape changes associated with development and maturation of the linear heart tube. o, outflow tract; $v$, ventricle. Arrowhead indicates failure of cardiomyocyte fusion at ventral midline. (E,F) CASZ1depleted embryos exhibit similar defects in cardiac looping and cardiac cell shape changes. $n=10$ per condition, two biological replicates. Scale bar: $50 \mu \mathrm{m}$.

between cell boundaries and accompanying breaks in the basement membrane of the myocardium (Fig. 4M-O, Fig. 5A-C). We further observed that the basement membrane protein laminin was ectopically deposited in the deeper layers of the myocardium in CHD5-depleted and in CASZ1-depleted hearts (Fig. 5D-I). Thus, CHD5 and CASZ1 function appear to establish or maintain myocardial tissue integrity.

To determine the onset of these cellular defects, we examined the expression of ZO-1 and another tight junction marker, claudin-5, at stage 33, a stage prior to any overt cardiac defect. We observed at these stages that ZO-1 (Fig. 6A-L) and claudin-5 (Fig. 6M-X) were expressed at low levels and in a diffuse pattern in CHD5- and CASZ1depleted embryos relative to controls (Fig. 6A-X). Furthermore, we did not detect any alterations in $\beta 1$-integrin expression between control and CASZ1 - or CHD5-depleted cardiac tissue, suggesting that the defects we observed were due to perturbations in tight junction formation and not to a general loss of extracellular matrix-cytoskeletal junctions (supplementary material Fig. S10). Taken together, these results suggest that proper tight junction formation and deposition of basement membrane between cardiomyocytes are dependent on CASZ1 and CHD5 at the period when these cells migrate and fuse at the ventral midline.

\section{CASZ1 activity requires interaction with CHD5}

Given the requirement for CASZ1 and CHD5 in cardiac development, we sought to determine further the relationship between CASZ1 and CHD5. We therefore assessed the requirement for CHD5 on expression of CASZ1. We observed that Casz1 expression appears unperturbed in CHD5-depleted embryos (supplementary material Fig. S11, data not shown) and found that CASZ1 nuclear localization in cardiomyocytes was maintained in CHD5-depleted hearts (supplementary material Fig. S11). Taken together, these results imply that CASZ1 does not function downstream of CHD5 and that CHD5 is not essential for CASZ1 nuclear localization.

To determine the relevance of the CASZ1-CHD5 interaction, we generated a deletion series of CASZ1 and found that CHD5 interacts within a previously uncharacterized region of the $\mathrm{C}$ terminus of CASZ1 (amino acids 785-998) (Fig. 7A,B). Our studies further showed that this region, henceforth referred to as the CHD5interacting domain (CID), is both necessary and sufficient for the CASZ1-CHD5 interaction (Fig. 7A,B).

To test whether CHD5 is required for the CASZ1-CHD5 interaction in the heart, we took advantage of previous findings that depletion of CASZ1 leads to cardiac defects manifested by failure of the heart to fuse across the midline, undergo correct looping and properly form cardiac chambers (Fig. 7C-H,O-Q) (Christine and Conlon, 2008). We further found that that these defects can at the least be partially rescued by mis-expression of fulllength CASZ1 mRNA (Fig. 7I-K,O-Q). However, mis-expression of mRNA encoding Caszl with a deletion of the CID [although expressed as determined by western blot analyses of embryos injected with Casz1 or Casz1 $1 \triangle C I D$ mRNA (supplementary material Fig. S12)] fails to rescue any aspect of heart development in CASZ1-depleted embryos (Fig. 7L-Q; supplementary material Table S1). Taken together, these results imply that the CHD5CASZ1 interaction is essential for CASZ1 function and heart morphogenesis.

To further assess the role of the CID domain of CASZ1, we explored further the observation that the global mis-expression of CASZ1 results in a cardiac phenotype similar to that of CHD5 depletion (Fig. 8A-H). One explanation for this observation is CASZ1 may bind and titer out endogenous CHD5. To test this possibility, we mis-expressed the CID domain of CASZ1 and found resulting embryos had phenotypes similar to that of mis-expression of fulllength CASZ1: cardiac looping and morphogenesis defects (CASZ1, $65 \%$; CID, 30\%) (Fig. 8E-L). Collectively, these findings support a role for the CASZ1-CHD5 interaction in heart development.

\section{DISCUSSION}

Our studies suggest a cooperative role for CHD5 and CASZ1 during cardiac development. We identify CHD5 as a CASZ1-interacting partner and establish that this functional interaction occurs via a novel uncharacterized region of CASZ1 that is necessary for proper cardiogenesis to proceed. Our data suggest that this interaction is required for cardiac morphogenesis during the early stages of cardiac looping and chamber formation, and that these defects are driven by a failure to establish proper cell-cell junctions and basement membrane deposition within myocardial tissue.

\section{CASZ1 and context-dependent transcriptional regulation}

CASZ1 was initially identified and characterized in Drosophila (dCas) as a transcriptional regulator of cellular fate within the developing central nervous system (Cui and Doe, 1992; Mellerick et al., 1992). $\mathrm{dCas}$ acts as a repressor and functions within a transcriptional cascade through the direct repression of $p d m$ (Kambadur et al., 1998), which 

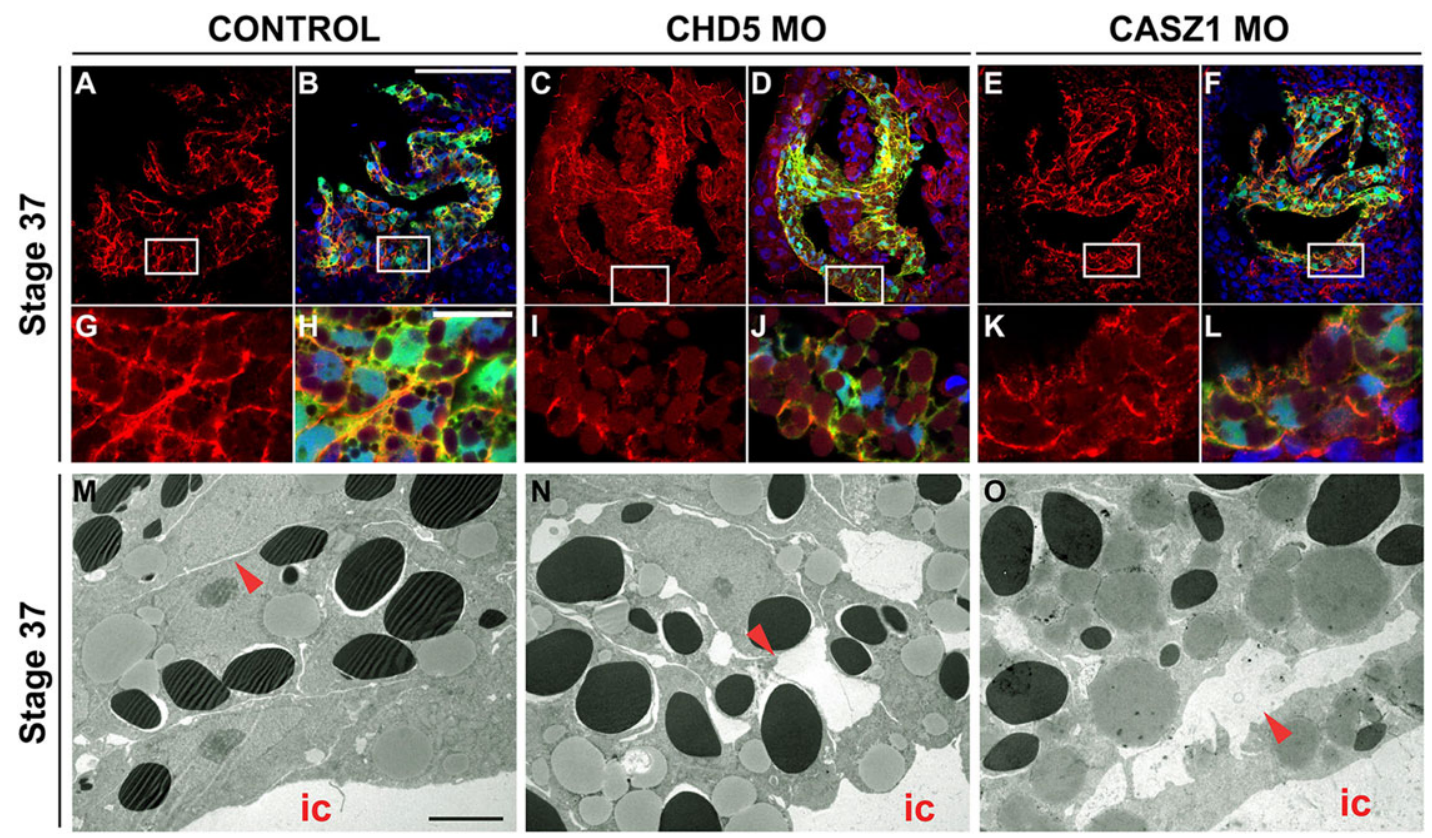

Fig. 4. CHD5 and CASZ1 are required for myocardial tissue adhesion. (A-L) Transverse sections of CHD5 and CASZ1-depleted CA-GFP embryos (stage 37) display diffuse or reduced expression of ZO-1, indicating defects in tight junction formation. Phenotypes represented across multiple sampled embryos ( $n \geq 4$, from two biological replicates). Boxes in A-F outline magnified images in G-L. Merge represents ZO-1 (red), DAPI (blue) and CA-GFP (green). (M-O) TEM imaging of transverse sections of control, CHD5-depleted and CASZ1-depleted myocardium (stage 37) reveal large intercellular gaps between cardiomyocytes (arrowheads) adjacent to the inner myocardial chamber (ic) in both CHD5-depleted and CASZ1-depleted embryos compared with control embryos ( $n \geq 2$, from two biological replicates). Scale bars: $100 \mu \mathrm{m}$ in A-F; $20 \mu \mathrm{m}$ in G-L; $5 \mu \mathrm{m}$ in M-O.

lacks a vertebrate homologue and does not appear to be an evolutionarily conserved target. Recent studies have provided further insight into neuronal-specific transcriptional targets of human CASZ1 (Liu et al., 2011b,c). With regards to cardiovascular development, recent studies have shown that in Xenopus and human epithelial cells, CASZ1 functions to directly regulate epidermal growth factor-like domain 7 (Egfl7), which in turn acts to promote RhoA-mediated vertebrate vascular development (Charpentier et al., 2013b). Taken together with the collective work in Drosophila, these studies imply that CASZ1 transcriptional activity is context dependent. However, the transcriptional mechanisms by which CASZ1 functions and the role of CHD5 in this regard remain to be established.

\section{CHD5 has distinct dual functions during development}

CHD5 was first identified as a nuclear-localized cardiac-expressed gene contained in a restricted region of chromosome 21 triplicated in individuals with DS (Egeo et al., 1998) and later found to localize to the nuclei of medaka fish cardiomyocytes (Murata et al., 2009). Interestingly, work on the yeast homolog of CHD5, MDM39, has identified synthetic interactions with Spflp, a P-type ATPase involved
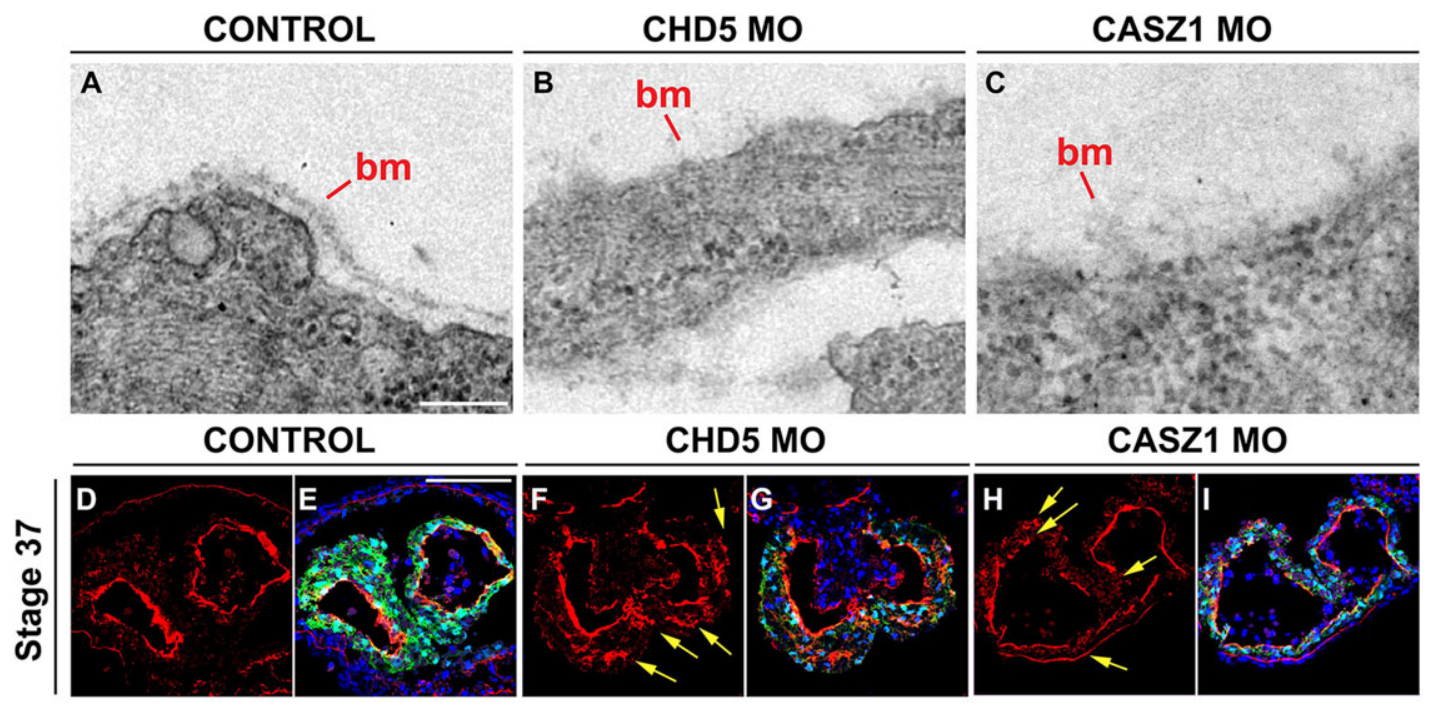

Fig. 5. CHD5 and CASZ1 are required for deposition of myocardial basement membrane. (A-C) The basement membrane (bm) forms as a discontinuous membrane on the basal surface of the myocardium in CHD5- and CASZ1-depleted embryos, as shown by TEM imaging (stage 37) (n=2). (D-I) CHD5- and CASZ1-depleted CA-GFP hearts (stage 37) exhibit areas of depleted or improperly localized laminin (yellow arrows). Phenotypes represented across multiple sampled embryos ( $n \geq 4$, from two biological replicates). Merge represents laminin (red), DAPI (blue) and CA-GFP (green). Scale bars: $0.2 \mu \mathrm{m}$ in A-C; $100 \mu \mathrm{m}$ in D-I. 

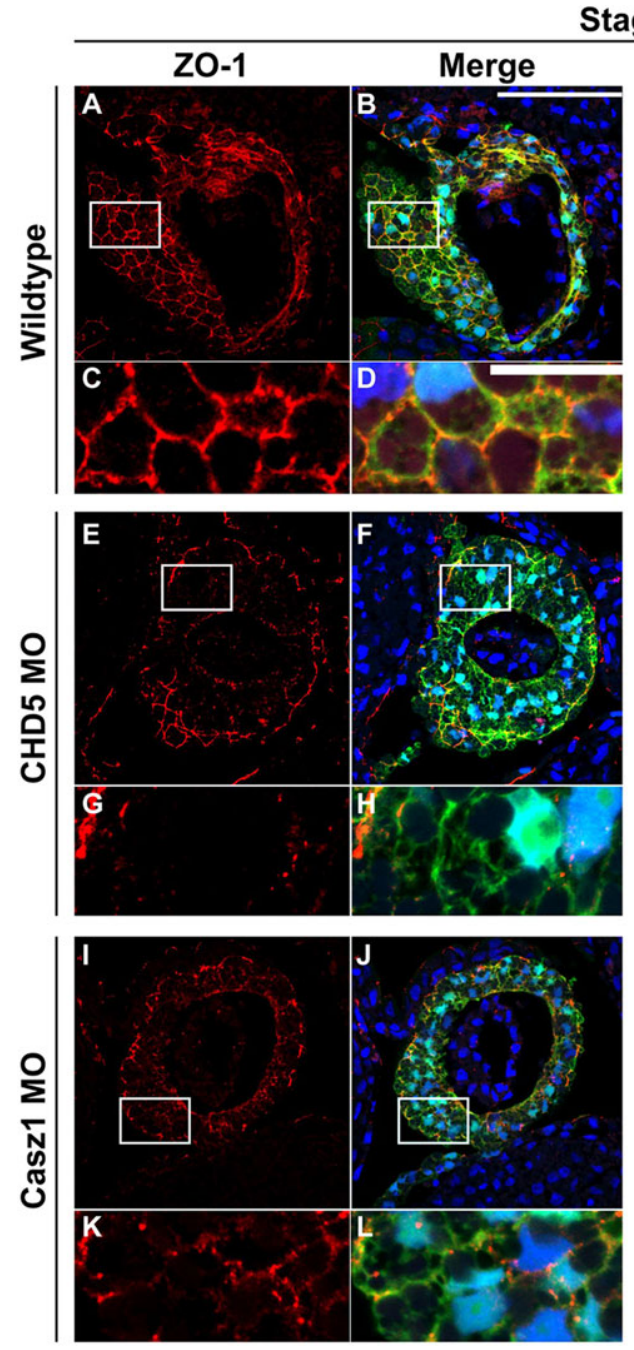

\section{Stage 33}
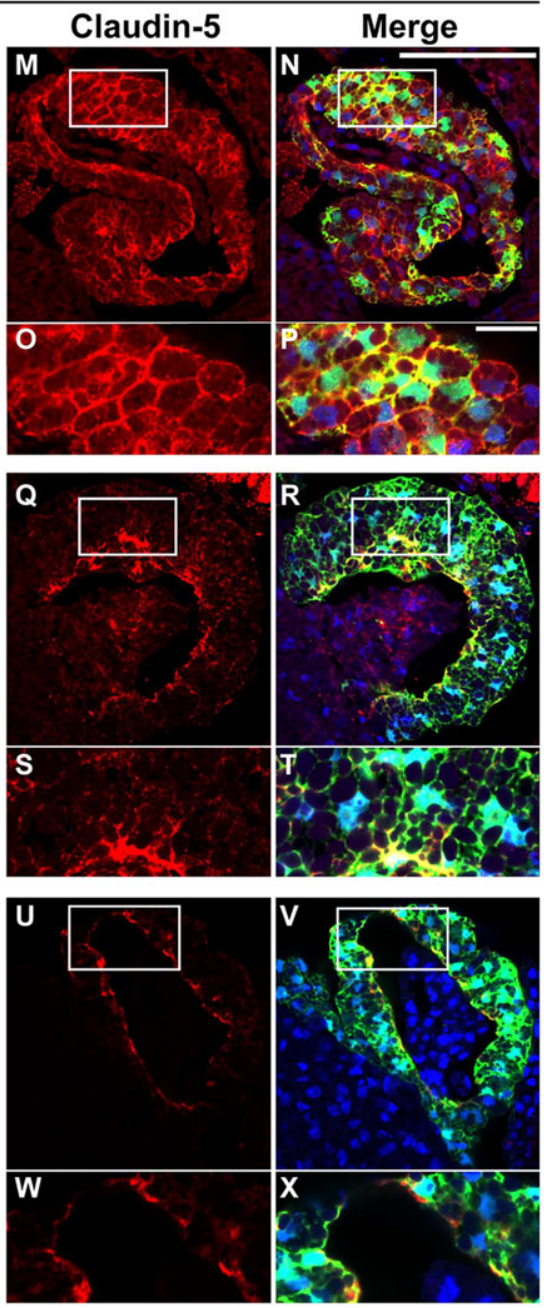

Fig. 6. CASZ1 and CHD5 are required for myocardial tissue adhesion prior to cardiac looping and chamber formation. (A-X) Control, CHD5-depleted and CASZ1-depleted CA-GFP embryos were examined for expression of the tight junction markers ZO-1 (A-L) and claudin-5 (M-X). Expression of both ZO-1 and claudin- 5 is reduced and appears diffuse and unorganized in transverse sections of CHD5- and CASZ1depleted hearts (stage 33). Phenotypes represented across multiple sampled embryos $(n \geq 4)$. White boxes in A,B,E,F,I,J,M,N,Q,R,U,V correspond to magnified images in $C, D, G, H, K, L$, $\mathrm{O}, \mathrm{P}, \mathrm{S}, \mathrm{T}, \mathrm{W}, \mathrm{X}$, respectively. Merge represents ZO-1 or claudin-5 (red), DAPI (blue) and CA-GFP (green). Scale bars: $100 \mu \mathrm{m}$ in A,B,E,F,I,J,M,N,Q, $\mathrm{R}, \mathrm{U}, \mathrm{V} ; 20 \mu \mathrm{m}$ in C,D, G,H,K,L,O,P,S,T,W,X. in protein transport of charged molecules across the ER membrane (Ando and Suzuki, 2005). These findings are supported by work demonstrating that, in mammalian cells, CHD5 can function as an ER membrane receptor that is responsible for the insertion of proteins into the ER membrane through a direct physical interaction with TRC40/ Asnal (Vilardi et al., 2011). Our studies demonstrate that CHD5 is nuclear localized along with CASZ1 in the developing myocardium, suggesting a cardiac-specific role in transcriptional regulation. Combined with past studies, our work raises the possibility that CHD5 has dual cellular functions: one CASZ1 dependent within the nucleus and the other CASZ1 independent in the ER membrane. The dual function of a protein in the nucleus and in the ER membrane is not unprecedented in biology: the transcription factor sterol regulatory element-binding protein (SREBP) has well-characterized roles in both the ER membrane and the nucleus during the processes of sterol synthesis (Espenshade and Hughes, 2007). CHD5 most likely interacts with distinct protein complexes, depending on its differing cellular roles. Future studies will determine the precise mechanisms that regulate the decision of CHD5 to localize in either the ER membrane or the nucleus during development, as well as whether CHD5 and CASZ1 interact and function within a larger transcriptional complex.

\section{CHD5 and Down's syndrome}

CHD in individuals with Down's syndrome encompasses a number of distinct disorders from tetralogy of Fallot to atrioventricular septal defects (Abbag, 2006). A recent clinical investigation determined that the disorder of chromosome 21 monosomy also results in cardiac defects, including hypoplastic and hypertrophied left ventricle and atrial as well as ventricular septal defects (Fisher et al., 2013). Recent work in mice investigating congenital heart disease in individuals with DS exclude Chd5 from the minimal region that induces congenital heart disease upon duplication (Liu et al., 2011a). By contrast, a recent screen in zebrafish identified CHD5 as a regulator of myocardial repolarization (Milan et al., 2009), and subsequent work in the medaka fish demonstrated that CHD5-depleted hearts had general looping and chamber defects (Murata et al., 2009). Our results demonstrate for the first time a mechanism by which CHD5 functions during cardiac development via interaction with a cardiac transcription factor and demonstrate cardiac defects in CHD5-depleted embryos. We demonstrate that CHD5 is crucial during development for the maintenance of proper cardiac morphogenesis and raise new questions about a possible link between CHD5, CASZ1 and congenital heart disease. CHD5 most likely possesses overlapping functional roles with other developmental genes within the chromosome 21 region that collectively are necessary at the correct dose for proper cardiac development to proceed. Continuing to characterize the link between CHD5, CASZ1 and cardiovascular disease will be crucial to understanding how this disease manifests not only in individuals with DS, but in the human population as a whole. 
A

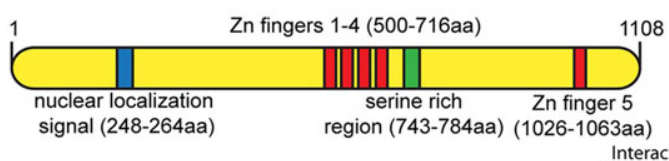

i. CASZ1

ii. CASZ1(1-998)

iii. CASZ1(1-828)

iv. CASZ1(561-1108)

v. CASZ1(561-998)

vi. CASZ1(785-998)

vii. CASZ1(828-998)

viii. CASZ1 $\triangle \mathrm{CID}$

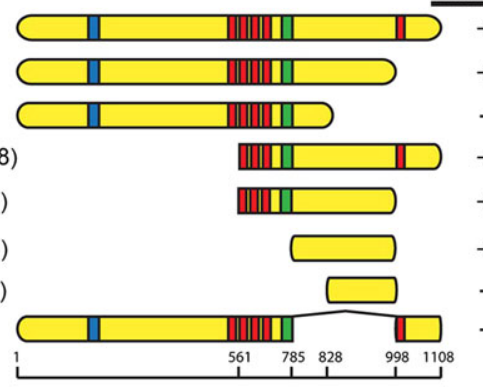

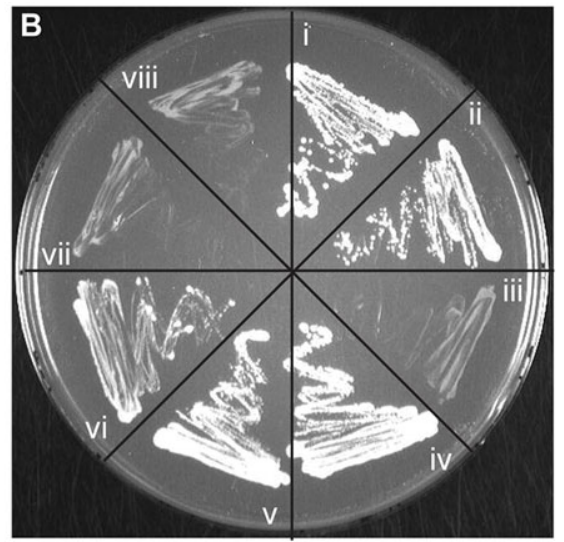
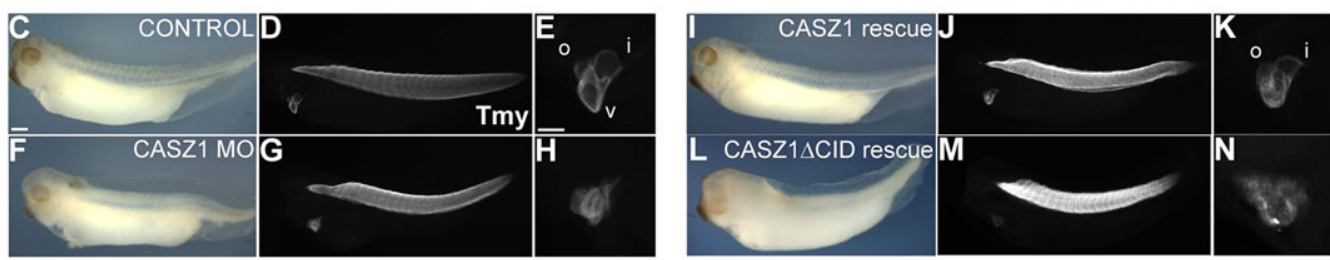

O $\%$ embryos displaying cardia bifida

$\mathbf{P}_{\%}$ embryos with proper chamber formation
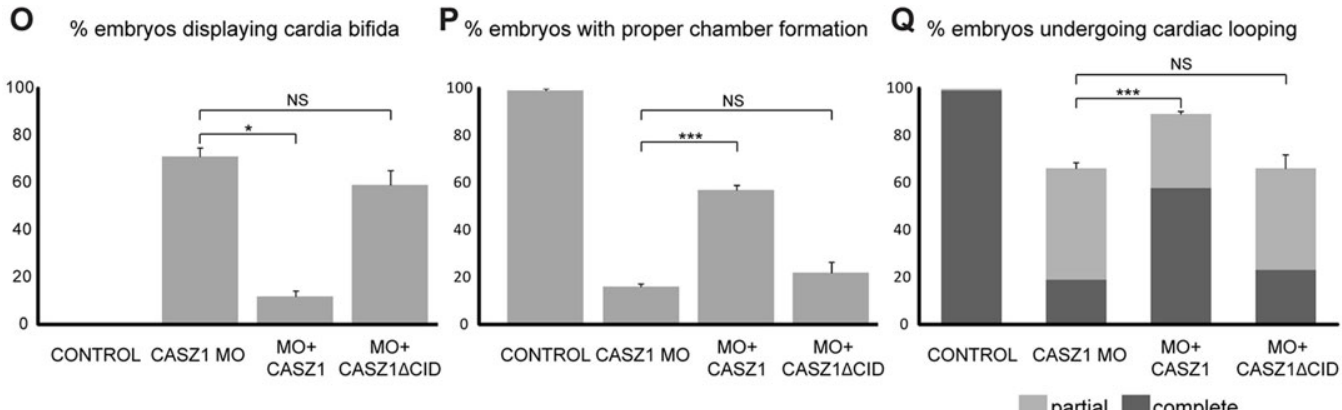

Fig. 7. CHD5 is crucial for CASZ1 function. (A,B) The region of CASZ1 containing the minimal CHD5-interacting domain amino acid residues 785-998 was identified using a yeast two-hybrid assay. (C-N) Control (C-E) and CASZ1-depleted (F-H) Xenopus embryos compared with CASZ1-depleted embryos coinjected with mRNA encoding either full-length CASZ1 (I-K) or CASZ1 $\triangle \mathrm{CID}(\mathrm{L}-\mathrm{N})$ at early tadpole stage (stage 37). Full-length CASZ1 partially rescued cardiac looping and chamber formation $(\mathrm{V})$, and outflow tract (o) and inflow tract (i) formation, whereas CASZ1 $\triangle$ CID failed to rescue any aspect of cardiogenesis. $(\mathrm{O}-\mathrm{Q})$ Three independent experiments $(n>20$ per condition) of embryos were scored for cardia bifida $(\mathrm{O})$, degree of proper cardiac looping $(\mathrm{P})$ and chamber formation $(Q)$ using whole-mount staining with anti-tropomyosin antibody. Fisher's exact test was performed for significance: NS, not significant; ${ }^{*} P=0.003$; ${ }^{* * *} P<0.0001$. Error bars represent calculated s.e.m. Scale bars: $500 \mu \mathrm{m}$ in C,D, F,G,I,J,L,M; $100 \mu \mathrm{m}$ in E,H,K,N.

\section{MATERIALS AND METHODS}

\section{cDNA library construction, yeast two-hybrid screen and} construction of CASZ1 mutants

Tissue containing the heart field was dissected from X. laevis embryos (stage 27-29) based on anatomical landmarks; i.e. cement gland and branchial arches. mRNA was purified with Ambion Poly(A) Purist mRNA purification kit, and cDNA was generated and cloned as prey into pGADT7-Rec in yeast strain AH109 using Matchmaker Two-Hybrid System (Clontech). Full-length CASZ1 (Christine and Conlon, 2008) (accession number NM_001110756) was cloned as bait into pGBKT7 in yeast strain Y187. Yeast strains were mated, and positive clones were detected on -Ade/-His/-Leu/-Trp plates (Clontech) containing $3 \mathrm{mM} 3$-amino-triazole according to the manufacturer's instructions. DNA from individual clones was isolated and sequenced. CASZ1 deletions were made using CASZ1-pGBKT7 bait vector as the parent vector, via fusion PCR mutagenesis. Primers and PCR conditions are provided in supplementary material Table S2.

\section{Whole-mount in situ hybridization}

Whole-mount in situ hybridization was conducted as described previously (Harland, 1991; Brown et al., 2003) using antisense probes for Nkx2.5 (Tonissen et al., 1994), Tbx20 (Brown et al., 2003), Caszl (Christine and Conlon, 2008), Chd5 (accession number BC094207), Eomes (accession number NM_001128652.1) and Otx2 (accession number BC077357) cloned in pSC-B-amp/kan (Stratagene). Embryos were cleared in 2:1 benzyl benzoate: benzyl alcohol and sectioned as previously described (Charpentier et al., 2013b).

\section{RT-PCR}

Primers were designed to $X$. laevis Chd5 (F, 5'-ATGGCGGAGAGCTGTGCGGC-3'; R, 5'-GTTTGTCGGTCATCTTGTTG-3') and X. laevis Casz1 (F, 5'-CTGGAGAATCACTGAGGCCA; R, 5'-CTCTGGTAGTTGCACTCAGG), and PCR was performed on cDNA derived from whole-embryo RNA at the indicated stages. EFlalpha was a positive control housekeeping gene. The efficiency of Chd5 splice-blocking morpholinos was tested by RT-PCR from cDNA derived from stage 25 whole embryos. Nested RTPCR was performed with primers to exon 1 (F, NMA-238: 5'-GTGCTTCTCTGCAATCTCTG), intron 2 (R, NMA-239: 5'-GGGATGTTGTTACCTCTTCC) and exon 2 (R, NMA-240: 5'-TGATAGTTGTGCCGTCCTGC). Wild-type cDNA and genomic DNA were used as positive controls for amplification by the designed primers.

\section{Morpholinos and Xenopus injections}

Antisense oligonucleotides against CHD5 (CCACGCACAGCTCTCCGCCATGTTT) were injected at $40 \mathrm{ng} / \mathrm{embryo}$ at the one-cell stage (Tandon et al., 2012). Embryos develop to the reported stages (Nieuwkoop and Faber, 1994). Efficiency of the morpholinos was tested by injection at the 

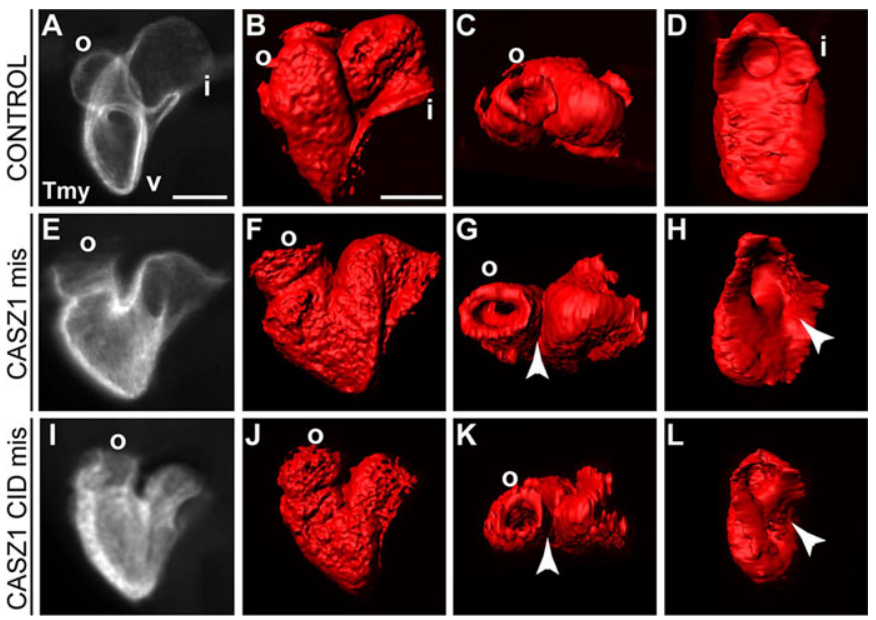

Fig. 8. The CASZ1 CID domain disrupts heart development. (A-D) Contro embryos at early tadpole stage (stage 37 ) exhibit looping and formation of the cardiac chambers. v, ventricle; o, outflow tract; i, inflow tract. (E-L) CASZ1 and CASZ1 CID misexpressing embryos display incomplete convergence of the outflow and inflow tract, as seen in dorsal views (l,K) (arrowheads), and a partially closed inflow tract, as seen in posterior views $(\mathrm{H}, \mathrm{L})$ (arrowheads). $n=23$ for CASZ1 mis; $n=29$ for CASZ1 CID mis; two biological replicates. Scale bars: $100 \mu \mathrm{m}$

one-cell stage with $40 \mathrm{ng}$ morpholino and subsequently injected in both blastomeres at the two-cell stage with full-length Xenopus CHD5-GFP mRNA (Open Biosystems, accession number BC094207). Western blot analysis at stage 27 was performed as previously published (Brown et al., $2005)$ with mouse anti-GFP $(1: 10,000$, JL- 8 , Clontech), mouse antiGAPDH as a positive loading control (1:10,000, MAB374, Millipore), HRP anti-mouse (1:10,000, 715-036-150, Jackson) and ECL Western Blotting Analysis System (Amersham). Additional antibodies used for immunoblotting include mouse anti-V5 (1:5000, R960CUS, Invitrogen), mouse anti-FLAG $(1: 1000$, F3165, Sigma), mouse anti-SHP2 $(1: 2500$, 610622, BD Transduction Laboratories), chick anti-CHD5 (1:10,000, ab51550, Abcam) and HRP anti-chick (1:30,000, 703-035-155, Jackson). Additional antisense oligonucleotides were designed against the exon 1 donor site (CGATGAATCGCTTACTTACAAGCGA) and the exon 2 donor site of CHD5 (CTGAAACCCGTTCACATTACCATGT). Exon 1 donor oligo and exon 2 donor oligo were injected separately at $40 \mathrm{ng}$ per embryo and collectively at a suboptimal combined $40 \mathrm{ng}$ per embryo (20 ng exon 1 donor oligo and $20 \mathrm{ng}$ exon 2 donor oligo).

For rescue experiments, cardiac actin-GFP (CA-GFP) transgenic Xenopus embryos (Latinkic et al., 2002) were injected at the one-cell stage with $80 \mathrm{ng}$ of CASZ1 morpholino (Christine and Conlon, 2008) and co-injected with $0.25 \mathrm{ng}$ of either CASZ1-V5 mRNA or CASZ1 $\Delta$ CID-V5 mRNA. Whole-mount immunohistochemistry was performed at stage 37 . Phenotypic scoring was statistically analyzed using a two-tailed Fisher's exact test for significance (three independent experiments with over 20 embryos per condition). For mis-expression experiments, embryos were injected at the one-cell stage with 2 ng full-length CASZ1 or CASZ1 CID (amino acids 785-998) mRNA. Embryos were collected for analysis at stage 37

\section{Immunohistochemistry}

Whole-mount antibody staining was performed as described previously (Goetz et al., 2006; Langdon et al., 2007, 2012) with mouse antitropomyosin (Developmental Studies Hybridoma Bank, 1:10) and Alexa 568 anti-mouse [1:250, $\operatorname{IgG}(\mathrm{H}+\mathrm{L})]$. Embryos were then cleared in 2:1 benzyl benzoate:benzyl alcohol and imaged on a Leica MZ16F fluorescence microscope with a Qimaging Retiga 4000RV Fast1394 camera with QCapture software as well as a Zeiss 700 confocal microscope with MyZen software. Z-stacks taken with MyZen software were imported to Imaris x64 6.1.5 software (Bitplane) for 3D rendering and isosurfacing at the University of North Carolina Microscopy Services Lab as previously described (Doherty et al., 2010). Sectioned antibody staining was performed with rabbit anti-CHD5 (Sigma, HPA018527, 1:100), guinea pig anti-CASZ1 (1:500) (Amin et al., 2014), mouse anti-ZO-1 (Invitrogen, 33-9100, 1:100), rabbit anti-laminin (Sigma, L9393, 1:100), rabbit anti-phospho-histone H3 (Millipore, 06-570, 1:100), rabbit anti-caspase 3 (Cell Signaling, 9661, 1:50), Alexa 647 donkey anti-rabbit (Invitrogen, A-31573, 1:1000), Alexa 546 donkey anti-mouse (Invitrogen, A10036, 1:250), Alexa 488 donkey anti-mouse $\operatorname{IgG}(\mathrm{H}+\mathrm{L}$; Invitrogen, A-21202, 1:1000) and Cy3 donkey anti-guinea pig (Jackson ImmunoResearch, 706165-148, 1:500). Stained sections were imaged on BX61 fluorescence microscope or Zeiss 700 confocal microscope, and ImageJ was used for analysis and cell counts. Cell counts of total cells positive for CA-GFP and total cells positive for phospho histone $\mathrm{H} 3$ expression were performed on transverse serial sections of control and CHD5-depleted stage 37 hearts $(n \geq 3)$. The mitotic index was calculated by dividing the total cells positive for CA-GFP and $\mathrm{pH} 3$ expression by the total cells positive for CA-GFP. Cell counts and mitotic index were compared for statistical significance using Student's $t$-test.

Embryos fixed in 4\% paraformaldehyde were embedded in 4\% low melt agarose (Promega) and sectioned by a Leica VT1200S vibratome at $100 \mu \mathrm{m}$. Sections were washed in PBS $+1 \%$ Triton X-100 (PBS-T), blocked in PBS$\mathrm{T}+10 \% \mathrm{FBS}$ and incubated overnight at $4^{\circ} \mathrm{C}$ with primary antibodies rabbit anti-claudin 5 (Santa Cruz, sc-28670, 1:500), mouse anti-ZO-1 (Invitrogen, 33-9100, 1:100), rabbit anti-fibronectin (Sigma, F3648, 1:100) and mouse anti- $\beta 1$ integrin (DSHB, 8C8, 1:100). The following day, sections were washed in PBS-T then incubated with secondary antibodies (1:1000 Alexa Fluor dyes, Molecular Probes) overnight at $4^{\circ} \mathrm{C}$. Sections were washed in PBS-T, incubated with $200 \mathrm{ng} / \mathrm{ml}$ DAPI (Sigma) and mounted. Images were taken with a Zeiss 700 confocal microscope.

\section{Electron microscopy imaging}

The pericardial cavity membrane was excised in embryos anesthetized in $0.1 \% \mathrm{w} / \mathrm{v}$ tricane in ice-cold $0.1 \times \mathrm{MBS}$ before being fixed in $2.5 \%$ glutaraldehyde (EM grade, Electron Microscopy Science) as previously reported (Tandon et al., 2013) in $0.1 \times \mathrm{MBS}(\mathrm{pH} 7.6)$ at $4^{\circ} \mathrm{C}$ overnight. Embryos were washed in $0.1 \times \mathrm{MBS}$, dehydrated in $100 \%$ ethanol, dried, mounted ventral side up and sputter coated with Gold-Palladium to approximately $40 \mathrm{~nm}$ thickness before being scanned with a Zeiss Supra 25 FESEM microscope. Embryos were also processed for sectioning and visualized on a Zeiss EM 910 transmission electron microscope (TEM) (Microscope Services Laboratory, UNC).

\section{Co-immunoprecipitation}

One-cell Xenopus embryos were injected with the following capped mRNAs: (1) $1 \mathrm{ng}$ Caszl-V5 only, (2) $1 \mathrm{ng} C h d 5-e G F P$ only, and (3) $1 \mathrm{ng}$ each Casz1-V5 and Chd5-eGFP. Embryos were collected at stage 12.5 and de-yolked as described previously (Link et al., 2006). Briefly, culture media was removed from embryos, and embryos were pipetted in de-yolking buffer $\left(55 \mathrm{mM} \mathrm{NaCl}, 1.8 \mathrm{mM} \mathrm{KCl}, 1.25 \mathrm{mM} \mathrm{NaHCO}_{3}\right.$ ) and mechanically disrupted by passing through a P1000 pipette tip inserted into a P200 pipette tip. Lysate was gently rocked at $4{ }^{\circ} \mathrm{C}$ for $2 \mathrm{~min}$, and centrifuged at 300 $\boldsymbol{g}$ for $30 \mathrm{~s}$ to gently pellet cells. Yolk-containing supernatant was discarded and pellet was resuspended in $1 \mathrm{ml}$ HEPES resuspension buffer [20 mM HEPES, $1.2 \%$ polyvinylpyrrolidone $(\mathrm{pH} 7.4), 1 \times$ protease inhibitors] and added dropwise to liquid nitrogen. Pellets were thawed and incubated in $5 \mathrm{ml}$ lysis buffer [200 mM K-HEPES ( $\mathrm{pH} 7.4$ ), $1.1 \mathrm{M} \mathrm{KOAc,} 20 \mathrm{mM}$ $\mathrm{MgCl}_{2}, 1 \%$ Tween $20,10 \mu \mathrm{M} \mathrm{ZnCl}_{2}, 10 \mu \mathrm{M} \mathrm{CaCl}_{2}, 500 \mathrm{mM} \mathrm{NaCl}, 1.0 \%$ Triton-X and $0.5 \%$ deoxycholic acid, $1 \times$ protease inhibitors]. Lysates were homogenized with 40 strokes each in a PTFE tissue grinder (VWR) and nuclei were further lysed with two $15 \mathrm{~s}$ pulses of a Polytron (Thermo Fisher Scientific) at setting 22, with 1 min recovery between pulses. Lysates were cleared at $7500 \mathrm{~g}$ for $10 \mathrm{~min}$. Once cleared, lysates were incubated with Dynabeads M-270 Epoxy (Invitrogen) that were coated with anti-GFP (Cristea et al., 2005) antibody. Incubation with antibody-conjugated beads was performed with end-over-end rotation at $4^{\circ} \mathrm{C}$ for $1 \mathrm{~h}$. Beads were washed seven times with lysis buffer and bound proteins were then eluted with $30 \mu 11 \times$ SDS sample buffer [ $10 \%$ glycerol, $100 \mathrm{mM}$ Tris ( $\mathrm{pH} 6.8$ ), $1 \%$ SDS, 5\% 2-mercaptoethanol, $10 \mu \mathrm{g} / \mathrm{ml}$ Bromophenol Blue]. Western blot 
analysis was used to confirm proper immunoprecipitation of CASZ1-V5 and CHD5-GFP with anti-V5 (46-1157, Invitrogen, 1:5000) and anti-GFP (JL8, Clontech, 1:10,000) antibodies, respectively.

\section{Acknowledgements}

We are extremely grateful to faculty of Microscopy Services Lab at UNC for microscopy help and also to lleana Cristea, Todd Greco and John Wallingford for helpful discussions and critical reading of manuscript. The antibody against tropomyosin (developed by Jim Jung-Ching Lin) was obtained from the Developmental Studies Hybridoma Bank, developed under the auspices of the $\mathrm{NICHD}$ and maintained by the University of lowa, Department of Biological Sciences, lowa City, IA 52242, USA.

Competing interests

The authors declare no competing financial interests.

\section{Author contributions}

As primary author, S.S. performed the majority of experiments and manuscript preparation. N.M.A., D.G. and M.S.C. contributed to experiments and to manuscript preparation and editing. K.S.C. contributed reagents and manuscript editing. F.L.C. contributed reagents, experiments, and manuscript editing and preparation.

\section{Funding}

This work is supported by grants to F.L.C. from The National Institutes of Health/The National Heart, Lung, and Blood Institute (NIH/NHLBI) [RO1 DE018825 and RO1 HL089641). S.S. and N.M.A. were supported by American Heart Association (AHA) awards. Deposited in PMC for release after 12 months.

\section{Supplementary material}

Supplementary material available online at

http://dev.biologists.org/lookup/suppl/doi:10.1242/dev.106518///DC1

\section{References}

Abbag, F. I. (2006). Congenital heart diseases and other major anomalies in patients with Down syndrome. Saudi Med. J. 27, 219-222.

Afouda, B. A. and Hoppler, S. (2009). Xenopus explants as an experimental model system for studying heart development. Trends Cardiovasc. Med. 19 220-226

Amin, N. M., Gibbs, D. and Conlon, F. L. (2014). Differential regulation of CASZ1 protein expression during cardiac and skeletal muscle development. Dev. Dyn. 243, 948-956.

Ando, A. and Suzuki, C. (2005). Cooperative function of the CHD5-like protein Mdm39p with a P-type ATPase Spf1p in the maintenance of ER homeostasis in Saccharomyces cerevisiae. Mol. Genet. Genomics 273, 497-506.

Bartlett, H. L. and Weeks, D. L. (2008). Lessons from the lily pad: using Xenopus to understand heart disease. Drug Discov. Today Dis. Models 5, 141-146.

Brade, T., Gessert, S., Kühl, M. and Pandur, P. (2007). The amphibian second heart field: Xenopus islet-1 is required for cardiovascular development. Dev. Biol. 311, 297-310.

Brown, D. D., Binder, O., Pagratis, M., Parr, B. A. and Conlon, F. L. (2003) Developmental expression of the Xenopus laevis Tbx20 orthologue. Dev. Genes Evol. 212, 604-607.

Brown, D. D., Martz, S. N., Binder, O., Goetz, S. C., Price, B. M. J., Smith, J. C. and Conlon, F. L. (2005). Tbx5 and Tbx20 act synergistically to control vertebrate heart morphogenesis. Development 132, 553-563.

Chaoui, R., Heling, K.-S., Sarioglu, N., Schwabe, M., Dankof, A. and Bollmann, R. (2005). Aberrant right subclavian artery as a new cardiac sign in second- and third-trimester fetuses with Down syndrome. Am. J. Obstet Gynecol. 192, 257-263.

Charpentier, M. S., Dorr, K. M. and Conlon, F. L. (2013a). Transcriptional regulation of blood vessel formation: the role of the CASZ1/Egfl7/RhoA pathway. Cell Cycle 12, 2165-2166.

Charpentier, M. S., Christine, K. S., Amin, N. M., Dorr, K. M., Kushner, E. J., Bautch, V. L., Taylor, J. M. and Conlon, F. L. (2013b). CASZ1 promotes vascular assembly and morphogenesis through the direct regulation of an EGFL7/RhoAmediated pathway. Dev. Cell 25, 132-143.

Christine, K. S. and Conlon, F. L. (2008). Vertebrate CASTOR is required for differentiation of cardiac precursor cells at the ventral midline. Dev. Cell 14 616-623.

Cristea, I. M., Williams, R., Chait, B. T. and Rout, M. P. (2005). Fluorescent proteins as proteomic probes. Mol. Cell. Proteomics 4, 1933-1941.

Cui, X. and Doe, C. Q. (1992). ming is expressed in neuroblast sublineages and regulates gene expression in the Drosophila central nervous system. Development 116, 943-952.
Doherty, J. T., Conlon, F. L., Mack, C. P. and Taylor, J. M. (2010). Focal adhesion kinase is essential for cardiac looping and multichamber heart formation. Genesis 48, 492-504.

Egeo, A., Mazzocco, M., Sotgia, F., Arrigo, P., Oliva, R., Bergonòn, S., Nizetic, D., Rasore-Quartino, A. and Scartezzini, P. (1998). Identification and characterization of a new human cDNA from chromosome 21q22.3 encoding a basic nuclear protein Hum. Genet. 102, 289-293.

Espenshade, P. J. and Hughes, A. L. (2007). Regulation of sterol synthesis in eukaryotes. Annu. Rev. Genet. 41, 401-427.

Evans, S. M., Yelon, D., Conlon, F. L. and Kirby, M. L. (2010). Myocardial lineage development. Circ. Res. 107, 1428-1444.

Fisher, D., DiPietro, A., Murdison, K. A. and Lemieux, C. A. (2013). Full monosomy 21: echocardiographic findings in the third molecularly confirmed case. Pediatr. Cardiol. 34, 733-735.

Gessert, S. and Kühl, M. (2009). Comparative gene expression analysis and fate mapping studies suggest an early segregation of cardiogenic lineages in Xenopus laevis. Dev. Biol. 334, 395-408.

Goetz, S. C. and Conlon, F. L. (2007). Cardiac progenitors and the embryonic cell cycle. Cell Cycle 6, 1974-1981.

Goetz, S. C., Brown, D. D. and Conlon, F. L. (2006). TBX5 is required for embryonic cardiac cell cycle progression. Development 133, 2575-2584.

Goodship, J., Cross, I., LiLing, J. and Wren, C. (1998). A population study of chromosome 22q11 deletions in infancy. Arc. Dis. Child. 79, 348-351.

Harland, R. M. (1991). In situ hybridization: an improved whole-mount method for Xenopus embryos. Methods Cell Biol. 36, 685-695.

Kaltenbrun, E., Tandon, P., Amin, N. M., Waldron, L., Showell, C. and Conlon, F. L. (2011). Xenopus: an emerging model for studying congenital heart disease. Birth Defects Res. A Clin. Mol. Teratol. 91, 495-510.

Kambadur, R., Koizumi, K., Stivers, C., Nagle, J., Poole, S. J. and Odenwald, W. F. (1998). Regulation of POU genes by castor and hunchback establishes layered compartments in the Drosophila CNS. Genes Dev. 12, 246-260.

Kolker, S. J., Tajchman, U. and Weeks, D. L. (2000). Confocal imaging of early heart development in Xenopus laevis. Dev. Biol. 218, 64-73.

Korbel, J. O., Tirosh-Wagner, T., Urban, A. E., Chen, X.-N., Kasowski, M., Dai, L., Grubert, F., Erdman, C., Gao, M. C., Lange, K. et al. (2009). The genetic architecture of Down syndrome phenotypes revealed by high-resolution analysis of human segmental trisomies. Proc. Natl. Acad. Sci. USA 106 12031-12036.

Langdon, Y. G., Goetz, S. C., Berg, A. E., Swanik, J. T. and Conlon, F. L. (2007) SHP-2 is required for the maintenance of cardiac progenitors. Development 134 4119-4130.

Langdon, Y., Tandon, P., Paden, E., Duddy, J., Taylor, J. M. and Conlon, F. L. (2012). SHP-2 acts via ROCK to regulate the cardiac actin cytoskeleton Development 139, 948-957.

Latinkić, B. V., Cooper, B., Towers, N., Sparrow, D., Kotecha, S. and Mohun, T. J. (2002). Distinct enhancers regulate skeletal and cardiac muscle-specific expression programs of the cardiac alpha-actin gene in Xenopus embryos. Dev. Biol. 245 $57-70$

Levy, D., Ehret, G. B., Rice, K., Verwoert, G. C., Launer, L. J., Dehghan, A., Glazer, N. L., Morrison, A. C., Johnson, A. D., Aspelund, T. et al. (2009) Genome-wide association study of blood pressure and hypertension. Nat. Genet. 41, 677-687.

Link, V., Shevchenko, A. and Heisenberg, C.-P. (2006). Proteomics of early zebrafish embryos. BMC Dev. Biol. 6, 1

Liu, Z., Yang, X., Tan, F., Cullion, K. and Thiele, C. J. (2006). Molecular cloning and characterization of human Castor, a novel human gene upregulated during cell differentiation. Biochem. Biophys. Res. Commun. 344, 834-844.

Liu, C., Morishima, M., Yu, T., Matsui, S.-I., Zhang, L., Fu, D., Pao, A., Costa, A. C. Gardiner, K. J., Cowell, J. K. et al. (2011a). Genetic analysis of Down syndromeassociated heart defects in mice. Hum. Genet. 130, 623-632.

Liu, Z., Naranjo, A. and Thiele, C. J. (2011b). CASZ1b, the short isoform of CASZ1 gene, coexpresses with CASZ1a during neurogenesis and suppresses neuroblastoma cell growth. PLOS ONE 6, e18557.

Liu, Z., Yang, X., Li, Z., McMahon, C., Sizer, C., Barenboim-Stapleton, L. Bliskovsky, V., Mock, B., Ried, T., London, W. B. et al. (2011c). CASZ1, a candidate tumor-suppressor gene, suppresses neuroblastoma tumor growth through reprogramming gene expression. Cell Death Differ. 18, 1174-1183.

Lyle, R., Béna, F., Gagos, S., Gehrig, C., Lopez, G., Schinzel, A., Lespinasse, J., Bottani, A., Dahoun, S., Taine, L. et al. (2009). Genotype-phenotype correlations in Down syndrome identified by array CGH in 30 cases of partial trisomy and partial monosomy chromosome 21. Eur. J. Hum. Genet. 17, 454-466.

Mellerick, D. M., Kassis, J. A., Zhang, S.-D. and Odenwald, W. F. (1992). castor encodes a novel zinc finger protein required for the development of a subset of CNS neurons in Drosophila. Neuron 9, 789-803.

Milan, D. J., Kim, A. M., Winterfield, J. R., Jones, I. L., Pfeufer, A., Sanna, S., Arking, D. E., Amsterdam, A. H., Sabeh, K. M., Mably, J. D. et al. (2009). Drug sensitized zebrafish screen identifies multiple genes, including GINS3, as regulators of myocardial repolarization. Circulation 120, 553-559.

Mohun, T. J., Leong, L. M., Weninger, W. J. and Sparrow, D. B. (2000). The morphology of heart development in Xenopus laevis. Dev. Biol. 218, 74-88. 
Murata, K., Degmetich, S., Kinoshita, M. and Shimada, E. (2009). Expression of the congenital heart disease 5/tryptophan rich basic protein homologue gene during heart development in medaka fish, Oryzias latipes. Dev. Growth Differ. 51, 95-107.

Newman, C. S. and Krieg, P. A. (1998). tinman-related genes expressed during heart development in Xenopus. Dev. Genet. 22, 230-238.

Nieuwkoop, P. D. and Faber, J. (1994). Normal Table of Xenopus Laevis (Daudin): A Systematical and Chronological Survey of the Development from the Fertilized Egg till the End of Metamorphosis. New York: Garland Publishing.

Pannese, M., Polo, C., Andreazzoli, M., Vignali, R., Kablar, B., Barsacchi, G. and Boncinelli, E. (1995). The Xenopus homologue of Otx2 is a maternal homeobox gene that demarcates and specifies anterior body regions. Development 121, 707-720.

Roger, V. L., Go, A. S., Lloyd-Jones, D. M., Adams, R. J., Berry, J. D., Brown, T. M., Carnethon, M. R., Dai, S., de Simone, G., Ford, E. S. et al. (2011). Heart disease and stroke statistics-2011 update: a report from the American Heart Association. Circulation 123, e18-e209.

Ryan, K., Butler, K., Bellefroid, E. and Gurdon, J. B. (1998). Xenopus eomesodermin is expressed in neural differentiation. Mech. Dev. 75, 155-158.

Shinohara, T., Tomizuka, K., Miyabara, S., Takehara, S., Kazuki, Y., Inoue, J., Katoh, M., Nakane, H., lino, A., Ohguma, A. et al. (2001). Mice containing a human chromosome 21 model behavioral impairment and cardiac anomalies of Down's syndrome. Hum. Mol. Genet. 10, 1163-1175.

Takeuchi, F., Isono, M., Katsuya, T., Yamamoto, K., Yokota, M., Sugiyama, T., Nabika, T., Fujioka, A., Ohnaka, K., Asano, H. et al. (2010). Blood pressure and hypertension are associated with 7 Loci in the Japanese population. Circulation 121, 2302-2309.

Tandon, P., Showell, C., Christine, K. and Conlon, F. L. (2012). Morpholino injection in Xenopus. Methods Mol. Biol. 843, 29-46.

Tandon, P., Miteva, Y. V., Kuchenbrod, L. M., Cristea, I. M. and Conlon, F. L. (2013). Tcf21 regulates the specification and maturation of proepicardial cells. Development 140, 2409-2421.

Tonissen, K. F., Drysdale, T. A., Lints, T. J., Harvey, R. P. and Krieg, P. A. (1994). $\mathrm{XNkx}-2.5$, a Xenopus gene related to $\mathrm{Nkx}-2.5$ and tinman: evidence for a conserved role in cardiac development. Dev. Biol. 162, 325-328.

Vacalla, C. M. H. and Theil, T. (2002). Cst, a novel mouse gene related to Drosophila Castor, exhibits dynamic expression patterns during neurogenesis and heart development. Mech. Dev. 118, 265-268.

Vilardi, F., Lorenz, H. and Dobberstein, B. (2011). WRB is the receptor for TRC40/ Asna1-mediated insertion of tail-anchored proteins into the ER membrane. J. Cell Sci. 124, 1301-1307.

Warkman, A. S. and Krieg, P. A. (2007). Xenopus as a model system for vertebrate heart development. Semin. Cell Dev. Biol. 18, 46-53. 